Journal of Computational and Applied Mechanics, Vol. 10, No. 1, (2015), pp. 65-82

DOI: $10.32973 /$ jcam.2015.005

\title{
BOUNDARY CONTOUR METHOD FOR MIXED BOUNDARY VALUE PROBLEMS IN THE DUAL SYSTEM OF PLANE ELASTICITY
}

\author{
SÁNDOR SZIRBIK \\ Institute of Engineering Mechanics, University of Miskolc \\ H-3515 Miskolc, Miskolc-Egyetemváros, Hungary \\ Sandor.Szirbik@uni-miskolc.hu
}

[Received: November 24, 2014, Accepted: January 30, 2015]

\begin{abstract}
In this paper the boundary contour method in the dual system of plane elasticity is developed further by clarifying the issue of how to apply the method to mixed boundary value problems on simply and multiply connected regions. In contrast to [1, [2] in which the contour is divided into two arcs on which tractions and displacements are imposed, respectively, here we shall allow the contour to be divided into an even number of arcs (the number of arcs should be at least four) assuming that tractions and displacements are prescribed on the subsequent arcs. This means that the equations of the boundary contour method set up in 2 are not sufficient to solve these mixed boundary value problems since they do not involve the supplementary conditions of single valuedness which should be kept in order for obtaining correct solutions. This paper presents the supplementary conditions of single valuedness and shows how to add them to the solution algorithm. Numerical examples prove the efficiency of this technique.
\end{abstract}

Mathematical Subject Classification: 74G05, 34B05

Keywords: Boundary contour method, the dual system of plane elasticity, supplementary conditions

\section{INTRODUCTION}

The paper presents a procedure which makes possible the application of the boundary contour method in the dual system of the plane elasticity to a class of the mixed boundary value problems.

The first paper devoted to the boundary contour method (BCM) in the primal system of two-dimensional linear elasticity was published in 1994 by A. Nagarjan at al. 3]. By observing that the integrand vector of the boundary element integrals without body forces is demonstrably divergence free in the primal system of the two and three-dimensional elasticity theory, the authors of $[3$ came to the conclusion that the numerical solution of three-dimensional problems requires the calculation of line integrals instead of surface integrals [4, while for planar problems evaluation 
of functions should be performed instead of calculating line integrals. The stress calculation established by this method is probably more accurate in many cases than that applied by the conventional boundary element method (BEM). This technique was developed further both for more $2 \mathrm{D}$ and for some $3 \mathrm{D}$ problems [5]. With this technique one can compute stresses and can solve shape optimization problems in two dimensions [6]. By using a hypersingular formulation, the accuracy of the stress components can be further increased at points on the contour 7].

The boundary integral equations of the direct method in the dual system of plane elasticity was formulated in 8 and $[9]$. In the dual system the first-order stress function and the rigid body rotation are the unknown functions, in contrast to the primal system of plane elasticity in which the fundamental variable is the displacement. The choice of first-order stress functions provides advantages in the stress computation and if the tractions are not continuous on the region's boundary. For multiply-connected regions or if there are two (or more) arcs subjected to tractions, supplementary conditions of single valuedness should be prescribed and satisfied - in this respect details are given in [10].

The boundary contour method in the dual system of plane elasticity is published in [1,2]. The divergence freedom in the dual system is based on the fact that the stress functions of order one and the rigid body rotation (the unknowns of problem) satisfy the field equations of plane elasticity. As a consequence of this condition, potential functions exist. Papers [1] and 2] publish the proof of the divergence freedom, present the appropriate potential functions assuming linear and quadratic approximations for the shape functions, and set up the discretized equations to prepare an algorithm for the numerical computations. Consequently, the greatest advantage of BCM in the dual system is that the computation of stresses on the boundary elements requires derivations only, that is, unlike conventional BEM, one can avoid the computation of singular integrals.

The present paper is organized into six sections. Section 2 is devoted to some preliminaries in which the direct method in the dual system of plane elasticity and the corresponding boundary conditions are shown assuming simple connected regions. In addition we have determined the corresponding shape functions in Section 3, provided that the approximation is quadratic. Section 4 deals with the issue of how to extend the dual boundary contour method for the class of boundary value problems in which the boundary is divided into even number of arcs (the number of arcs should be at least four) provided that displacements or tractions are prescribed on the subsequent arcs. We shall clarify that the integrand in the conditions of single valuedness is also divergence free in the dual system of plane elasticity and shall determine the corresponding shape functions assuming quadratic approximation. In addition we present the conditions of single valuedness in a form discretized appropriately for the algorithm of computations. Section 5 contains some simple numerical examples. The last section is the conclusion. Some earlier results which are utilized in the paper are gathered in the Appendix. 


\section{Preliminaries}

Throughout this paper $x_{\rho}$ are rectangular Cartesian coordinates referred to an origin $O$. Greek subscripts are assumed to have the range $(1,2)$. In accordance with the notations introduced $\delta_{\rho \lambda}$ is the Kronecker symbol, $\partial_{\rho}$ stands for the derivatives with respect to $x_{\rho}$ and $\epsilon_{\rho \pi 3}$ is the permutation symbol.

Consider a simply connected inner region $\mathcal{A}_{i}$ and its supplementary exterior region $\mathcal{A}_{e}$. The common contour $\mathcal{L}_{o}$ of the regions can be divided into two parts denoted by $\mathcal{L}_{t}$ and $\mathcal{L}_{u}$. We shall assume that $\left[\mathcal{L}_{t}\right]\left\{\mathcal{L}_{u}\right\}$ is the union of those arcs on which [stress functions (arcs subjected to tractions)] \{strain boundary conditions (derivatives of displacements with respect to the arc coordinate) $\}$ are imposed. If the number of arcs on the boundary is four they are denoted by $\mathcal{L}_{t 1}, \mathcal{L}_{t 3}$ and $\mathcal{L}_{u 2}, \mathcal{L}_{u 4}$, respectively.
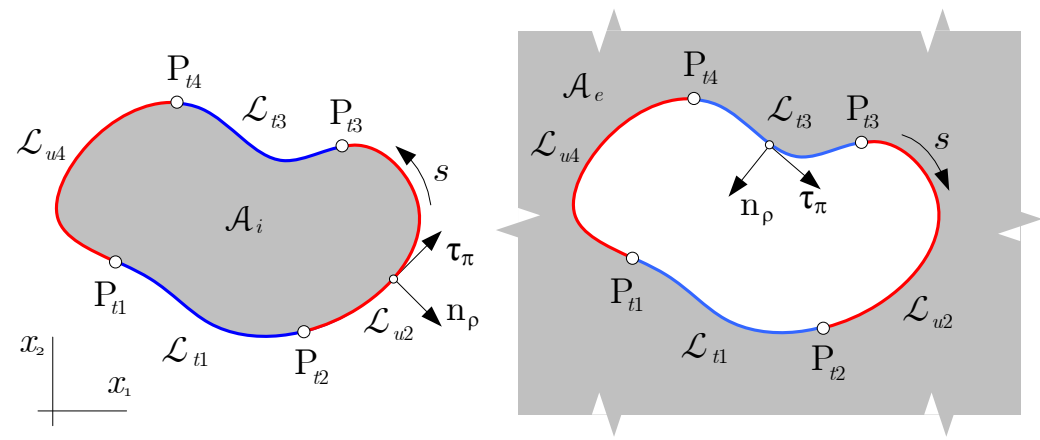

Figure 1. The inner region $\mathcal{A}_{i}$ and the exterior region $\mathcal{A}_{e}$

Let $Q$ and $M$ be two points in the region $\mathcal{A}_{i}$ or $\mathcal{A}_{e}$ (the source point and the point of effect). We shall assume temporarily that the point $Q$ is fixed.

The integral equation (the second dual Somigliana formula) to be solved is of the form:

$$
\begin{array}{r}
c_{\kappa \lambda}(Q) \mathfrak{u}_{\lambda}(Q)=\tilde{\mathfrak{u}}_{\kappa}(Q)+\oint_{\mathcal{L}_{o}} \mathfrak{U}_{\kappa \lambda}(M, Q) \mathfrak{t}_{\lambda}(M) \mathrm{d} s-\oint_{\mathcal{L}_{o}} \mathfrak{T}_{\kappa \lambda}(M, Q) \mathfrak{u}_{\lambda}(M) \mathrm{d} s \\
Q, M \in \mathcal{L}_{o},
\end{array}
$$

where $\mathfrak{u}_{\lambda}(Q)$ is the stress function vector, $\mathfrak{t}_{\lambda}(M)$ stands for the derivative $-d u_{\lambda} / d s$ $\left(u_{\lambda}\right.$ denotes the displacements, $s$ is the arc coordinate on the contour $\mathcal{L}_{o}$ ), the points $Q$ and $M$ are taken on the contour $\mathcal{L}_{o}, \tilde{\mathfrak{u}}_{\kappa}(Q)$ is the stress function vector that gives a constant stress state at infinity, $\mathfrak{U}_{\kappa \lambda}(M, Q)$ and $\mathfrak{T}_{\kappa \lambda}(M, Q)$ are the fundamental solutions of order one and two - see Appendix A and $\left[9\right.$ for details - and $c_{\kappa \lambda}(Q)=$ $\delta_{\kappa \lambda} / 2$ if the contour is smooth at $Q$, otherwise it depends on the angle formed by the tangents to the contour at $Q$. The above integral equation is that of the direct method in the dual system of elasticity with unknowns $\mathfrak{u}_{\lambda}(M)$ on $\mathcal{L}_{u}$ and $\mathfrak{t}_{\lambda}(M)$ on $\mathcal{L}_{t}$. We remark that the two line integrals in (2.1) should be taken in principal value. 
As the distance between the points $Q$ and $M$ tends to zero, weak (logarithmic) and strong singularity appear in the integrands of formula (2.1). We can avoid computation of strongly singular integrals if we assume that $\mathfrak{u}_{\lambda}(Q)=\mathfrak{u}_{\lambda}(M)=$ constant on the whole boundary. Under this condition $\mathfrak{t}_{\lambda}(M)=0$. In other words there are no stresses due to constant stress functions. On the basis of all that has been said equation (2.1) yields

$$
c_{\kappa \lambda}(Q) \mathfrak{u}_{\lambda}(Q)=\oint_{\mathcal{L}_{o}} \mathfrak{T}_{\kappa \lambda}(M, Q) \mathfrak{u}_{\lambda}(Q) \mathrm{d} s \quad Q, M \in \mathcal{L}_{o} .
$$

Subtracting equation 2.2 from 2 , we obtain the regularized form

$$
0=\tilde{\mathfrak{u}}_{\kappa}(Q)+\underbrace{\oint_{\mathcal{L}_{o}}\left\{\mathfrak{U}_{\kappa \lambda}(M, Q) \mathfrak{t}_{\lambda}(M)-\mathfrak{T}_{\kappa \lambda}(M, Q)\left[\mathfrak{u}_{\lambda}(M)-\mathfrak{u}_{\lambda}(Q)\right]\right\} \mathrm{d} s}_{I_{\kappa}(Q)} \quad Q, M \in \mathcal{L}_{o} .
$$

In this way we have eliminated the tensor $c_{\kappa \lambda}(Q)$ and the strong singularity from equation 2.1.

We have proved [1] that the integrand in formula $\sqrt{2.3}$ is divergence free. Consequently there exists a potential function $\phi_{\kappa}(M, Q)$. After factoring out the outward normal $n_{\rho}(M)$ from the integrand on the right-hand side in 2.3 , the coefficient of $n_{\rho}(M)$ is denoted, for brevity, by $P_{\kappa \rho}(M, Q)$. Let $M_{1}$ and $M_{3}$ be two points on the contour for which $s_{1}<s_{3}$. Taking now the line integral between the points $M_{1}$ and $M_{3}$ and making use of the above results we have

$$
\int_{M_{1}}^{M_{3}} P_{\kappa \rho}(M, Q) n_{\rho}(M) \mathrm{d} s=\phi_{\kappa}\left(M_{3}, Q\right)-\phi_{\kappa}\left(M_{1}, Q\right) .
$$

Assume that the contour $\mathcal{L}_{o}$ is divided into $n_{b e}$ boundary elements. We regard the points $M_{1}$ and $M_{3}$ if they were the two extremities of an element on the boundary. Then integrating element by element we get

$$
I_{\kappa}(Q)=\sum_{e=1}^{n_{b e}}\left[\phi_{\kappa}^{e}\left(M_{3}, Q\right)-\phi_{\kappa}^{e}\left(M_{1}, Q\right)\right]
$$

where the superscript $e$ shows that $\phi_{\kappa}$ is taken on the $e$-th element.

If the region under consideration is the exterior one $\mathcal{A}_{e}$, the regularized integral equation (2.3) assumes the form

$$
0=\tilde{\mathfrak{u}}_{\kappa}(Q)+I_{\kappa}(Q) .
$$

The results considered above make it possible to establish the boundary contour method in the dual system of plane elasticity by using linear and quadratic approximations [1,2].

Equation (2.3) should be associated with appropriate boundary conditions. One can readily check that the stress function vector (the dual displacement vector) on 
the $\operatorname{arcs} \mathcal{L}_{t i}$ is of the form

$$
\hat{\mathfrak{u}}_{\rho}(s)=\int_{P_{t i}}^{s} \hat{t}_{\rho}(\sigma) \mathrm{d} \sigma \quad s \in \mathcal{L}_{t i} \quad i=1,3,
$$

where a hat on the letters denotes the values prescribed. Consequently, equation

$$
\mathfrak{u}_{\rho}(s)=\hat{\mathfrak{u}}_{\rho}(s)+C_{\rho}^{(t i)} \quad s \in \mathcal{L}_{t i} \quad i=1,3
$$

is equivalent to the traction boundary condition. Observe that the quantities $C_{\rho}^{(t i)}$ in this equation are undetermined constants of integration.

The strain boundary conditions on the arcs of $\mathcal{L}_{u i}$ have the following form:

$$
\frac{\mathrm{d} \hat{u}_{\lambda}}{\mathrm{d} s}=n_{\rho}\left[\epsilon_{\rho \pi 3} e_{\pi \lambda}-\delta_{\rho \lambda} \varphi_{3}\right] \quad s \in \mathcal{L}_{u i} \quad i=2,4,
$$

where $\hat{u}_{\lambda}(s)$ is the displacement vector prescribed on $\mathcal{L}_{u i}$, the in-plane components of strain are denoted $e_{\kappa \lambda}$ and $\varphi_{3}$ stands for the rigid-body rotation.

If tractions (stress functions) are imposed on the whole contour $\mathcal{L}_{o}\left(\mathcal{L}_{t} \equiv \mathcal{L}_{o}\right)$ the compatibility condition in the large

$$
\int_{\mathcal{L}_{o}} n_{\rho}\left[\epsilon_{\rho \pi 3} e_{\pi \lambda}-\delta_{\rho \lambda} \varphi_{3}\right] \mathrm{d} s=0
$$

should also be satisfied. If tractions (stress functions) are imposed only on the arcs $\mathcal{L}_{t 1}, \mathcal{L}_{t 3}$ of the contour $\mathcal{L}_{o}$, the supplementary conditions of single valuedness to be satisfied assume the form

$$
\int_{\mathcal{L}_{t i}} n_{\rho}\left[\epsilon_{\rho \pi 3} e_{\pi \lambda}-\delta_{\rho \lambda} \varphi_{3}\right] \mathrm{d} s-\left.\hat{u}_{\lambda}\right|_{P_{t i}} ^{P_{t, i+1}}=0 \quad i=1,3,
$$

where the extremities of the $\operatorname{arc} \mathcal{L}_{t i}$ are denoted by $P_{t i}$ and $P_{t, i+1}$, respectively.

If the compatibility field equations

$$
\epsilon_{\kappa \rho 3} e_{\lambda \kappa} \partial_{\rho}+\varphi_{3} \partial_{\lambda}=\epsilon_{\kappa \rho 3}\left(e_{\lambda \kappa}-\epsilon_{\lambda \kappa 3} \varphi_{3}\right) \partial_{\rho}=0
$$

are fulfilled then the supplementary compatibility conditions 2.11 are not independent - one can be omitted 11].

In this way we have as many independent conditions of single valuedness (2.11) as there are undetermined integration constants $C_{\rho}^{(t i)}$ in the traction boundary condition 2.8. We can also set two constants (one vector $C_{\rho}^{(t i)}$ ) to zero since no stresses belong to constant stress functions - see [10,11] for details. Papers [1,2] deal with simply connected regions assuming that the contour is divided into two arcs: displacements are prescribed in one arc and tractions on the other. For this reason there is no need to imply the supplementary conditions of single valuedness into the model. If there are, however, more than two arcs subjected to tractions, we have no choice but to involve the supplementary conditions of single valuedness in the equations system to make it determined. 


\section{Shape And potential FUnCtions}

Assume that the contour $\mathcal{L}_{o}$ is divided into $n_{\text {be }}$ boundary elements and a boundary element $e$ has five nodal points. The first, third, and fifth nodal points are denoted by $M_{1}, M_{2}$ and $M_{3}$ the second and fourth by $K_{1}$ and $K_{2}$. Over the element and its neighborhood we shall approximate the fundamental variables $\mathfrak{u}_{\lambda}$ and $\varphi_{3}$. Observe that, according to the strain boundary conditions 2.9 , the displacement derivative $\mathfrak{t}_{\lambda}(M)$ in equation 2.3 requires the knowledge of the rotation $\varphi$. In order to obtain appropriate approximations, the vector of the fundamental variables $\left[\mathfrak{u}_{l}\right]^{T}=\left[\mathfrak{u}_{1}\left|\mathfrak{u}_{2}\right|\right.$ $\left.\varphi_{3}\right]$ should satisfy the basic equation (see equation (3.3) in 9 for details). We have selected the approximation

$$
\left[\begin{array}{c}
\mathfrak{u}_{1} \\
\mathfrak{u}_{2} \\
-\varphi_{3}
\end{array}\right]^{e}=\left[\begin{array}{c}
a_{1}^{e}+a_{2}^{e} x_{1}+a_{3}^{e} x_{2}-2 a_{7}^{e} x_{1} x_{2}+a_{8}^{e} x_{1}^{2}+a_{9}^{e} x_{2}^{2} \\
a_{4}^{e}+a_{5}^{e} x_{1}-a_{2}^{e} x_{2}+a_{7}^{e} x_{2}^{2}-2 a_{8}^{e} x_{1} x_{2}+a_{10}^{e} x_{1}^{2} \\
a_{6}^{e}+K a_{7}^{e} x_{2}+K a_{8}^{e} x_{1}+K a_{9}^{e} x_{1}+K a_{10}^{e} x_{2}
\end{array}\right]
$$

where $K=(1-\nu) / \mu$. This approximation satisfies the basic equation. The constants

$$
\left[\mathbf{a}^{e}\right]^{T}=\left[\begin{array}{llllllllll}
a_{1}^{e} & a_{2}^{e} & a_{3}^{e} & a_{4}^{e} & a_{5}^{e} & a_{6}^{e} & a_{7}^{e} & a_{8}^{e} & a_{9}^{e} & a_{10}^{e}
\end{array}\right]
$$

in 3.2 are related to the ten physical quantities

$$
\left[\mathbf{p}^{e}\right]^{T}=\left[\mathfrak{u}_{1}^{M_{1}}\left|\mathfrak{u}_{2}^{M_{1}}\right| \mathfrak{t}_{1}^{K_{1}}\left|\mathfrak{t}_{2}^{K_{1}}\right| \mathfrak{u}_{1}^{M_{2}}\left|\mathfrak{u}_{2}^{M_{2}}\right| \mathfrak{t}_{1}^{K_{2}}\left|\mathfrak{t}_{2}^{K_{2}}\right| \mathfrak{u}_{1}^{M_{3}} \mid \mathfrak{u}_{2}^{M_{3}}\right]
$$

taken on the element $e$ via the equation

$$
\underbrace{\mathbf{p}^{e}}_{(10 \times 1)}=\underbrace{\mathbf{T}^{e}}_{(10 \times 10)} \underbrace{\mathbf{a}^{e}}_{(10 \times 1)},
$$

where the transformation matrix $\mathbf{T}^{e}$ - see Appendix B.2 in [12 for details - depends only on the nodal coordinates and the outward unit normals at $K_{1}$ and $K_{2}$. It can be proved that transformation (3.4) is one to one if the nodal points are different.

A new local coordinate system $\left(\eta_{1}, \eta_{2}\right)$ centered at the point $M_{1}$ is introduced and its axes $\left(\eta_{1}, \eta_{2}\right)$ are parallel to the global ones. If we approximate the vector $\mathfrak{u}_{k}$ in equation (3.1) in the local coordinate system $\left(\eta_{1}, \eta_{2}\right)$ by quadratic functions over the elements we get the following vector:

$$
\left[\begin{array}{c}
\mathfrak{u}_{1} \\
\mathfrak{u}_{2} \\
-\varphi_{3}
\end{array}\right]^{e}=\left[\begin{array}{c}
\hat{a}_{1}^{e}+\hat{a}_{2}^{e} \eta_{1}+\hat{a}_{3}^{e} \eta_{2}-2 a_{7}^{e} \eta_{1} \eta_{2}+a_{8}^{e} \eta_{1}^{2}+a_{9}^{e} \eta_{2}^{2} \\
\hat{a}_{4}^{e}+\hat{a}_{5}^{e} \eta_{1}-\hat{a}_{2}^{e} \eta_{2}+a_{7}^{e} \eta_{2}^{2}-2 a_{8}^{e} \eta_{1} \eta_{2}+a_{10}^{e} \eta_{1}^{2} \\
\hat{a}_{6}^{e}+K a_{7}^{e} \eta_{2}+K a_{8}^{e} \eta_{1}+K a_{9}^{e} \eta_{1}+K a_{10}^{e} \eta_{2}
\end{array}\right],
$$

in which

$$
\begin{aligned}
& \hat{a}_{1}^{e}=a_{1}^{e}+a_{2}^{e} x_{1}+a_{3}^{e} x_{2}-2 a_{7}^{e} x_{1} x_{2}+a_{8}^{e} x_{1}^{2}+a_{9}^{e} x_{2}^{2}, \hat{a}_{2}^{e}=a_{2}^{e}-2 a_{7}^{e} x_{2}+2 a_{8}^{e} x_{1}, \\
& \hat{a}_{3}^{e}=a_{3}^{e}-2 a_{7}^{e} x_{1}+2 a_{9}^{e} x_{2}, \hat{a}_{4}^{e}=a_{4}^{e}+a_{5}^{e} x_{1}-a_{2}^{e} x_{2}+a_{7}^{e} x_{2}^{2}-2 a_{8}^{e} x_{1} x_{2}+a_{10}^{e} x_{1}^{2}, \\
& \hat{a}_{5}^{e}=a_{5}^{e}-2 a_{8}^{e} x_{2}+2 a_{10}^{e} x_{1}, \hat{a}_{6}^{e}=a_{6}^{e}+K a_{7}^{e} x_{2}+K a_{8}^{e} x_{1}+K a_{9}^{e} x_{1}+K a_{10}^{e} x_{2} .
\end{aligned}
$$

By

$$
\left[\hat{\mathbf{a}}^{e}\right]^{T}=\left[\begin{array}{llllllllll}
\hat{a}_{1}^{e} & \hat{a}_{2}^{e} & \hat{a}_{3}^{e} & \hat{a}_{4}^{e} & \hat{a}_{5}^{e} & \hat{a}_{6}^{e} & a_{7}^{e} & a_{8}^{e} & a_{9}^{e} & a_{10}^{e}
\end{array}\right]
$$


we denote the vector of constants in the local system. For the relation between a and â one can write

$$
\underbrace{\hat{\mathbf{a}}^{e}}_{(10 \times 1)}=\underbrace{\mathbf{B}^{M_{1}}}_{(10 \times 10)} \underbrace{\mathbf{a}^{e}}_{(10 \times 1)},
$$

where the transformation matrix $\mathbf{B}^{M_{1}}$ depends only on the coordinates $x_{1}$ and $x_{2}$ of the point $M_{1}$ - see 1,2 for details. Consequently we obtain a relation of the form

$$
\underbrace{\hat{\mathbf{a}}^{e}}_{(10 \times 1)}=\underbrace{\mathbf{B}^{M_{1}}}_{(10 \times 10)} \underbrace{\left(\mathbf{T}^{e}\right)^{-1}}_{(10 \times 10)} \underbrace{\mathbf{p}^{e}}_{(10 \times 1)}
$$

which relates the physical quantities to the weight parameters.

After approximating vector $\mathfrak{u}_{k}$ in the local coordinate system $\left(\eta_{1}, \eta_{2}\right)$ over an element we arrive at a linear combination of the linearly independent shape vectors

$$
\begin{aligned}
& {\left[{ }^{1} \mathfrak{u}_{k}\right]^{T}=\left[\begin{array}{lll}
1 & 0 & 0
\end{array}\right], \quad\left[{ }^{6} \mathfrak{u}_{k}\right]^{T}=\left[\begin{array}{lll}
0 & 0 & 1
\end{array}\right],} \\
& {\left[{ }^{2} \mathfrak{u}_{k}\right]^{T}=\left[\begin{array}{lll}
\eta_{1} & -\eta_{2} & 0
\end{array}\right], \quad\left[{ }^{7} \mathfrak{u}_{k}\right]^{T}=\left[\begin{array}{lll}
-2 \eta_{1} \eta_{2} & \eta_{2}^{2} & K \eta_{2}
\end{array}\right],} \\
& {\left[{ }^{3} \mathfrak{u}_{k}\right]^{T}=\left[\begin{array}{lll}
\eta_{2} & 0 & 0
\end{array}\right], \quad\left[{ }^{8} \mathfrak{u}_{k}\right]^{T}=\left[\begin{array}{lll}
\eta_{1}^{2} & -2 \eta_{1} \eta_{2} & K \eta_{1}
\end{array}\right],} \\
& {\left[{ }^{4} \mathfrak{u}_{k}\right]^{T}=\left[\begin{array}{lll}
0 & 1 & 0
\end{array}\right], \quad\left[{ }^{9} \mathfrak{u}_{k}\right]^{T}=\left[\begin{array}{lll}
\eta_{2}^{2} & 0 & K \eta_{1}
\end{array}\right],} \\
& {\left[{ }^{5} \mathfrak{u}_{k}\right]^{T}=\left[\begin{array}{lll}
0 & \eta_{1} & 0
\end{array}\right], \quad\left[{ }^{10} \mathfrak{u}_{k}\right]^{T}=\left[\begin{array}{lll}
0 & \eta_{1}^{2} & K \eta_{2}
\end{array}\right],}
\end{aligned}
$$

which satisfies the basic equation in [9]. Later on, it will be advantageous to choose always the current source point $Q$ for the center of the local coordinate system.

By substituting these shape vectors into

$$
\begin{array}{r}
{ }^{i} \phi_{\kappa}\left(\eta_{1}, \eta_{2}\right)=\int \epsilon_{\rho \pi 3}\left\{\mathfrak{U}_{\kappa \lambda}\left(\eta_{1}, \eta_{2}\right){ }^{i} \mathfrak{t}_{\lambda \rho}\left(\eta_{1}, \eta_{2}\right)-\mathfrak{T}_{\kappa \lambda \rho}\left(\eta_{1}, \eta_{2}\right){ }^{i} \mathfrak{u}_{\lambda}\left(\eta_{1}, \eta_{2}\right)\right\} \mathrm{d} \eta_{\pi} \\
i=1, \ldots, 10
\end{array}
$$

and performing the integrals for each shape function one can set up the corresponding potential functions ${ }^{i} \phi_{\kappa}$ in a closed form. The matrix $\mathfrak{T}_{\kappa \lambda \rho}\left(\eta_{1}, \eta_{2}\right)$ in equation (3.11) is given in Appendix A. The potential functions ${ }^{i} \phi_{\kappa}$ which belong to the regularized dual Somigliana formula (2.3) are also presented in Appendix B.

Fulfillment of the boundary element equations is enforced only at the points $M_{1}$, $M_{2}$ and $M_{3}$ of the elements. Assume that the collocation point $Q$ coincides with the first nodal point of the $h$-th element. Further for the stress functions at this point we introduce the notations $\mathfrak{u}_{1}(Q)=\hat{a}_{1}^{h}$ and $\mathfrak{u}_{2}(Q)=\hat{a}_{4}^{h}$ in the local coordinate system. With these notations, we can write

$$
\underbrace{\left[\mathbf{u}^{e}(M)\right]-\left[\mathbf{u}^{h}\left(Q_{j}\right)\right]}_{(2 \times 1)}=\underbrace{\mathbf{U}^{e}\left(\eta_{1}, \eta_{2}\right)}_{(2 \times 10)} \underbrace{\tilde{\mathbf{a}}^{e}}_{(10 \times 1)},
$$

where $\left[\mathbf{u}^{e}(M)\right]^{T}=\left[\mathfrak{u}_{1}^{e}(M) \mid \mathfrak{u}_{2}^{e}(M)\right]$ is the vector of stress functions at the point $M$ of the $e$-th element and the columns of the matrix $\mathbf{U}^{e}\left(\eta_{1}, \eta_{2}\right)$ are constituted by 
the vectors ${ }^{i} \mathfrak{u}_{\kappa}(i=1, \ldots, 10)$. In this way the vector of constants $\tilde{\mathbf{a}}^{e}$ can now be rewritten as:

$$
\begin{gathered}
{\left[\tilde{\mathbf{a}}^{e}\right]^{T}=\left[\begin{array}{cccccccccc}
\beta_{1}^{e} & \hat{a}_{2}^{e} & \hat{a}_{3}^{e} & \beta_{4}^{e} & \hat{a}_{5}^{e} & \hat{a}_{6}^{e} & a_{7}^{e} & a_{8}^{e} & a_{9}^{e} & a_{10}^{e}
\end{array}\right],} \\
\beta_{1}^{e}=\hat{a}_{1}^{e}-\hat{a}_{1}^{h}, \quad \begin{array}{c}
\beta_{4}^{e}=\hat{a}_{4}^{e}-\hat{a}_{4}^{h} .
\end{array}
\end{gathered}
$$

The potential functions $\phi_{11}$ and $\phi_{24}$ have singularity if the point of effect $M$ approaches the source point. In this case $\hat{a}_{1}^{e}=\hat{a}_{1}^{j}$ and $\hat{a}_{4}^{e}=\hat{a}_{4}^{j}$, therefore $\beta_{1}^{e}=\beta_{4}^{e}=0$, and so the evaluation of these singular potential functions can be avoided. Fulfillment of the regularized form of the boundary element equation $\sqrt{2.3}$ is enforced only at the points $M_{1}, M_{2}$ and $M_{3}$ of elements. Turning to global numbering we denote these points by $Q_{j}$ where $j=1, \ldots, 2 n_{b e}$. Hereafter, the point $Q_{j}$ is called nodal point. As is shown in 1, 2, the regularized dual Somigliana formula 2.3 can be rewritten into the discretized form

$$
\sum_{e=1}^{n_{b e}} \underbrace{\boldsymbol{\Phi}^{j e} \mathbf{B}^{j}\left(\mathbf{T}^{e}\right)^{-1}}_{\mathbf{N}^{j e}} \mathbf{p}^{e}=\tilde{\mathbf{u}}(\infty) \quad j=1, \ldots, 2 n_{b e},
$$

where $\boldsymbol{\Phi}^{j e}$ is a matrix with size $(2 \times 10)$ that are formed by the differences of the potential functions ${ }^{i} \phi_{\kappa}$ between the end points of the $e$-th boundary element, the transformation matrix $\mathbf{T}^{e}$ - in accordance with all that has been said so far - depends only on the nodal coordinates and the outward unit normal at $K_{j}$ and the transformation matrix $\mathbf{B}^{j}$ depends only on the global coordinates $Q_{j}$. If the region under consideration is an exterior one, the right side is not zero but is equal to the stress function representation $\tilde{\mathbf{u}}(\infty)$ of the stresses at infinity. If the contour is divided into only two arcs on which tractions and displacements are imposed, then the equation system (3.14) is solvable, otherwise too many undetermined constants of integration remain in (2.8).

\section{Compatibility CONDitions IN DiscRetized FORM}

This means that the equations of the boundary contour method in the dual system [1,2 are to be supplemented with further conditions of single valuedness which are needed for determining the integration constants $C_{\rho}^{(t i)}$. However, as has already been mentioned, one supplementary condition of single valuedness should not be fulfilled since two constants (one of the vectors $C_{\rho}^{(t i)}$ ) can be set to zero. In this section we shall show how to add the necessary supplementary conditions of single valuedness to the solution algorithm.

One can prove with ease that the integral in the compatibility conditions 2.10 , 2.11 is divergence free if the stress function vector $\mathfrak{u}_{\lambda}(M)$ and the corresponding rotation $\varphi_{3}(M)$ fulfill the field equations. This means that these quantities are derived from the fundamental solution. It is also clear that the tensor $e_{\kappa \pi \lambda}(M, Q)-$ which is in fact a strain tensor - is obtained from the fundamental solution of order one via the dual kinematic equation

$$
t_{\kappa \lambda}=\epsilon_{\kappa \rho 3} \mathfrak{u}_{\lambda} \partial_{\rho}+\stackrel{o}{t_{\kappa \lambda}}
$$




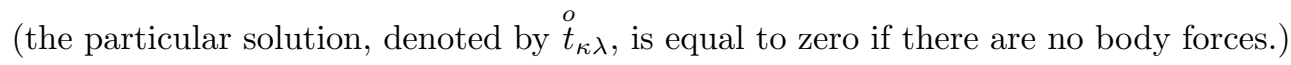
and Hooke's law

$$
e_{\kappa \lambda}=\frac{1}{2 \mu}\left(t_{\kappa \lambda}-\nu t_{\psi \psi} \delta_{\kappa \lambda}\right)
$$

( $\mu$ is the shear modulus of elasticity, $\nu$ is the Poisson number), in a such way that we substitute the tensor $\mathfrak{U}_{\kappa \lambda}(M, Q)$ for the vector $\mathfrak{u}_{\lambda}(M)$ and the corresponding rotation $\varphi_{3}(M)$ for $\mathfrak{U}_{\kappa 3}(M, Q)$ as follows:

$$
e_{\kappa \pi \lambda}(M, Q)=\frac{1}{2 \mu}\left(\epsilon_{\pi \rho 3} \mathfrak{U}_{\kappa \lambda} \partial_{\rho}-\nu \epsilon_{\psi \rho 3} \mathfrak{U}_{\kappa \psi} \partial_{\rho} \delta_{\pi \lambda}\right)
$$

After substitution of equation 4.3 in the compatibility condition in the large 2.10 , we get the following line integral:

$$
\int_{\mathcal{L}_{o}} n_{\rho}(M)\left[\epsilon_{\rho \pi 3} e_{\kappa \pi \lambda}(M, Q)-\delta_{\rho \lambda} \mathfrak{U}_{\kappa 3}(M, Q)\right] \mathrm{d} s=0 \quad Q, M \in \mathcal{L}_{o} .
$$

If we apply Gauss's theorem, the integral on the left hand side can be transformed into an integral taken over $\mathcal{A}_{i}$ :

$$
\int_{\mathcal{A}_{i}}^{M} \stackrel{\partial}{\partial}_{\rho}\left[\epsilon_{\rho \pi 3} e_{\kappa \pi \lambda}(M, Q)-\delta_{\rho \lambda} \mathfrak{U}_{\kappa 3}(M, Q)\right] \mathrm{d} A=0 .
$$

Observe that the term in square brackets in equation 4.5 is nothing but the dual balance equation since the quantities are derived from the fundamental solution $\mathfrak{U}_{\kappa \lambda}(M, Q)$. Consequently, the derivative of the expression with respect to $\eta_{\rho}$ is identically equal to zero. By using this condition, we arrive at the potential functions

$$
{ }^{i} \psi_{\kappa}\left(\eta_{1}, \eta_{2}\right)=\int \epsilon_{\rho \pi 3}\left[\epsilon_{\rho \lambda 3}{ }^{i} e_{\lambda \kappa}\left(\eta_{1}, \eta_{2}\right)-\delta_{\rho \kappa}{ }^{i} \varphi_{3}\left(\eta_{1}, \eta_{2}\right)\right] \mathrm{d} \eta_{\pi}
$$

which belong again to the weight parameters $a_{i}$. The corresponding potential functions ${ }^{i} \psi_{\kappa}$ have been calculated by making use of equation 4.6 for each ${ }^{i} e_{\lambda \kappa}$ and ${ }^{i} \varphi_{3}$ - the former two quantities can be calculated from the vectors ${ }^{i} \mathfrak{u}_{k}(i=1, \ldots, 10)$. Hence we have set up the corresponding potential functions ${ }^{i} \psi_{\kappa}$ in a closed form. The potential functions ${ }^{i} \psi_{\kappa}$ are given by:

$$
\begin{aligned}
& { }^{1} \psi_{1}=0, \quad{ }^{1} \psi_{2}=0, \\
& { }^{6} \psi_{1}=\eta_{2}, \\
& { }^{6} \psi_{2}=-\eta_{1}, \\
& { }^{2} \psi_{1}=-\frac{1}{2 \mu} \eta_{2}, \\
& { }^{2} \psi_{2}=-\frac{1}{2 \mu} \eta_{1}, \\
& { }^{7} \psi_{1}=\frac{2-\nu}{2 \mu} \eta_{2}^{2} \\
& { }^{7} \psi_{2}=\frac{\nu}{\mu} \eta_{1} \eta_{2}, \\
& { }^{3} \psi_{1}=\frac{1}{2 \mu}(1-\nu) \eta_{1}, \quad{ }^{3} \psi_{2}=-\frac{\nu}{2 \mu} \eta_{2}, \\
& { }^{8} \psi_{1}=-\frac{\nu}{\mu} \eta_{1} \eta_{2} \\
& { }^{8} \psi_{2}=-\frac{2-\nu}{2 \mu} \eta_{1}^{2}, \\
& { }^{4} \psi_{1}=0, \\
& { }^{4} \psi_{2}=0 \text {, } \\
& { }^{9} \psi_{1}=\frac{1-\nu}{\mu} \eta_{1} \eta_{2} \\
& { }^{9} \psi_{2}=-\frac{1-\nu}{2 \mu} \eta_{1}^{2} \\
& { }^{5} \psi_{1}=\frac{\nu}{2 \mu} \eta_{1}, \\
& { }^{5} \psi_{2}=-\frac{1-\nu}{2 \mu} \eta_{2}, \\
& { }^{10} \psi_{1}=\frac{1-\nu}{2 \mu} \eta_{2}^{2}, \\
& { }^{10} \psi_{2}=-\frac{1-\nu}{\mu} \eta_{1} \eta_{2} .
\end{aligned}
$$

With the knowledge of the functions ${ }^{i} \psi_{\kappa}$ one can handle the line integrals in the supplementary condition of single valuedness in the same way as the other line integrals in the integral equation (2.1), i.e. by using potential functions. Let $M_{1}$ and $M_{3}$ 
be two points on the contour for which $s_{1}<s_{3}$. Considering now the line integral between the points $M_{1}$ and $M_{3}$ and making use of the above results we have

$$
\int_{M_{1}}^{M_{3}} n_{\rho}(M)\left[\epsilon_{\rho \lambda 3} e_{\lambda \kappa}(M, Q)-\delta_{\rho \kappa} \mathfrak{u}_{3}(M)\right] \mathrm{d} s=\psi_{\kappa}\left(M_{3}, Q\right)-\psi_{\kappa}\left(M_{1}, Q\right) \quad M \in \mathcal{L}_{t i} .
$$

The actual position of $Q$ has no influence on the result of integral (4.7), however, it is advised to choose the position of $Q$ so that it should be out of the arc $\mathcal{L}_{t i}$.

The number of arcs on which tractions are prescribed is denoted by $n_{t}$. It is clear from Figure 1 that now we have two arcs (denoted by $\mathcal{L}_{t 1}, \mathcal{L}_{t 3}$ ) with prescribed tractions. Hence $n_{t}=2$. Here and in the sequel the extremities of arcs $\mathcal{L}_{t 1}, \mathcal{L}_{t 3}$ are assumed to coincide with some nodal points on $\mathcal{L}_{o}$. The regularized form of the boundary conditions can be written on one of these two arcs as

$$
\sum_{e \in \mathcal{L}_{t i}} \underbrace{\mathbf{\Psi}^{2 n_{b e}+j, e} \mathbf{B}^{2 n_{b e}+j}\left(\mathbf{T}^{e}\right)^{-1}}_{\mathbf{M}^{2 n_{b e}+j, e}} \mathbf{p}^{e}=\hat{\mathbf{u}}^{2 n_{b e}+j} \quad j=1, \ldots, 2\left(n_{t}-1\right),
$$

where $\Psi^{j e}$ is a matrix with size $(2 \times 10)$. The elements of this matrix are the differences of the potential functions ${ }^{i} \psi_{\kappa}$ between the end points of the $e$-th boundary element, while $\hat{\mathbf{u}}^{2 n_{b e}+j}(j=1,2)$ denotes the difference of displacements at the extremities of the $\operatorname{arc} \mathcal{L}_{t i}$ :

$$
\hat{\mathbf{u}}^{2 n_{b e}+j}=\left[\begin{array}{c}
\hat{u}_{1}\left(P_{t, 2 i}\right) \\
\hat{u}_{2}\left(P_{t, 2 i}\right)
\end{array}\right]-\left[\begin{array}{c}
\hat{u}_{1}\left(P_{t, 2 i-1}\right) \\
\hat{u}_{2}\left(P_{t, 2 i-1}\right)
\end{array}\right] \quad i=1 \text { or } 2 .
$$

Making use of equations (3.14 and 4.8 and exploiting that the stress functions are continuous at the extremities of the elements, one can construct an equation system. However, we eliminate the continuity of the stress functions at those points which coincide with some extremities of the $\operatorname{arcs} \mathcal{L}_{t 1}, \mathcal{L}_{t 3}$. Accordingly, the corresponding columns in matrices $\mathbf{N}^{j e}$ and $\mathbf{M}^{j e}$ can be added to each other. The continuity conditions that the elements of the matrices $\mathbf{p}^{e}$ should meet are also to be taken into account. Under these conditions, we obtain the equations

$$
\mathbf{K} \mathbf{f}=\left[\begin{array}{c}
\mathbf{0} \\
\hat{\mathbf{u}}
\end{array}\right] \quad \text { for the inner region and } \quad \mathbf{K} \mathbf{f}=\left[\begin{array}{c}
\tilde{\mathbf{u}} \\
\hat{\mathbf{u}}
\end{array}\right] \quad \text { for the outer region } .
$$

Here $\mathbf{K}$ is a matrix with size $\left(4 n_{b e}+2\left(n_{t}-1\right)\right) \times\left(8 n_{b e}+2\left(n_{t}-1\right)\right)$ and $\mathbf{f}$ denotes the vector of physical quantities. The columns of the matrix $\mathbf{K}$ that are multiplied by the prescribed quantities should be grouped on the right side of the equation in order to obtain the equation system to be solved. As the approximation we have chosen satisfies the basic equation in 9], the given displacement derivatives already determine the integration constants $C_{\rho}^{(\overrightarrow{t \imath})}$ in the traction boundary condition on each curve separately.

After solving the equations system (4.10, we have the nodal values of the unknown stress functions and the unknown displacement derivatives on the boundary curve, that is, every element of the vector $\mathbf{p}^{e}$ is known. The first dual Somigliana formula 
for the outer region to be solved is of the form:

$$
\mathfrak{u}_{\kappa}(Q)=\tilde{\mathfrak{u}}_{\kappa}(Q)+\oint_{\mathcal{L}_{o}} \mathfrak{U}_{\kappa \lambda}(M, Q) \mathfrak{t}_{\lambda}(M) \mathrm{d} s-\oint_{\mathcal{L}_{o}} \mathfrak{T}_{k \lambda}(M, Q) \mathfrak{u}_{\lambda}(M) \mathrm{d} s \quad Q \in \mathcal{A}_{e} .
$$

For an inner region, $\tilde{\mathfrak{u}}_{\kappa}(Q)$ is zero in the previous equation. The formula (4.11) can be rewritten - in accordance with that has been seen earlier - into a discretized form with the notations introduced. In this way, one can compute the stress functions $\mathfrak{u}_{\lambda}(Q)$ at an arbitrary point from the following equation:

$$
\mathbf{u}(Q)=\tilde{\mathbf{u}}(\infty)+\sum_{e=1}^{n_{b e}} \boldsymbol{\Phi}^{Q e} \mathbf{B}^{Q}\left(\mathbf{T}^{e}\right)^{-1} \mathbf{p}^{e}
$$

where the transformation matrix $\mathbf{B}^{Q}$ (see Appendix $\mathrm{C}$ for details) and $\boldsymbol{\Phi}^{Q e}$ depend on the coordinates of the source point position. Derivatives of the stress functions follow from equation 4.12 taken at the source point $Q$. Hence

$$
\frac{\partial}{\partial x_{\kappa}} \mathbf{u}(Q)=\frac{\partial}{\partial x_{\kappa}} \tilde{\mathbf{u}}(\infty)+\sum_{e=1}^{n_{b e}}\left[\boldsymbol{\Phi}^{Q e}\left(\frac{\partial}{\partial x_{\kappa}} \mathbf{B}^{Q}\right)-\left(\frac{\partial}{\partial \eta_{\kappa}} \boldsymbol{\Phi}^{Q e}\right) \mathbf{B}^{Q}\right]\left(\mathbf{T}^{e}\right)^{-1} \mathbf{p}^{e}
$$

According to the dual kinematic equations (4.1) we can determine the stresses by using the following equations:

$$
\begin{gathered}
\sigma_{11}(Q)=\sigma_{11}(\infty)+\left.\frac{\partial \mathfrak{u}_{1}}{\partial x_{2}}\right|_{Q}, \quad \sigma_{22}(Q)=\sigma_{22}(\infty)-\left.\frac{\partial \mathfrak{u}_{2}}{\partial x_{1}}\right|_{Q}, \\
\tau_{12}(Q)=\tau_{12}(\infty)-\left.\frac{\partial \mathfrak{u}_{2}}{\partial x_{2}}\right|_{Q}=\tau_{21}(Q)=\tau_{21}(\infty)+\left.\frac{\partial \mathfrak{u}_{1}}{\partial x_{1}}\right|_{Q} .
\end{gathered}
$$

\section{NumERICAL EXAMPLES}

Problem 1. The first problem is a benchmark to test the discretized form of the supplementary conditions of single valuedness in the calculation algorithm. We consider a circular region with radius $R=10 \mathrm{~mm}$. The material properties are $\mu=8 \cdot 10^{4} \mathrm{MPa}$, $\nu=0.3$. On the $\operatorname{arcs} \mathrm{AB}(\varphi \in[0, \pi / 2])$ and $\mathrm{CD}(\varphi \in[\pi, 1.5 \pi])$ of the contour the normal stress is $\sigma_{o}=100 \mathrm{MPa}$ and there is no shear stress. On the $\operatorname{arcs} \mathrm{BC}(\varphi \in[\pi / 2, \pi])$ and DA $(\varphi \in[1.5 \pi, 2 \pi])$ of the contour

$$
u_{o}=\frac{(1-2 \nu) \sigma_{o} R}{2 \mu}
$$

is the radial displacement and there is no displacement in the circumferential direction - see Figure 2. The supplementary conditions of single valuedness are imposed on the 


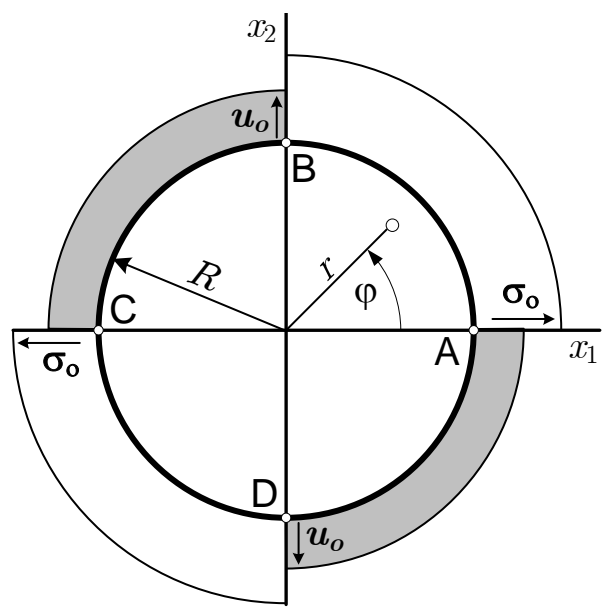

Figure 2. Circular region with two arcs subjected to tractions

arc CD and the difference between the displacements at the extremities of this arc is determined by the formula for the radial displacement. The exact solutions for this problem are given by the equations

$$
\begin{array}{rlrl}
\mathfrak{u}_{1} & =\sigma_{\mathrm{o}} r \sin \varphi, & \mathfrak{u}_{2} & =-\sigma_{\mathrm{o}} r \cos \varphi, \\
\mathfrak{t}_{1} & =\frac{1-2 \nu}{2 \mu} \sigma_{\mathrm{o}} \sin \varphi, & \mathfrak{t}_{2}=-\frac{1-2 \nu}{2 \mu} \sigma_{\mathrm{o}} \cos \varphi,
\end{array}
$$

where $r$ and $\varphi$ are polar coordinates. One can check with ease that these solutions determine a homogeneous state of stress. At the internal points the exact solutions for the stresses are as follows: $\sigma_{11}=\sigma_{22}=\sigma_{\mathrm{o}}=100[\mathrm{MPa}], \tau_{12}=0$. Table 1 below represents the numerical results obtained at various internal points.

Table 1: Solutions for stress components

\begin{tabular}{|c|c|c|c|c|}
\hline$x_{1}[\mathrm{~mm}]$ & $x_{2}[\mathrm{~mm}]$ & $\sigma_{11}[\mathrm{MPa}]$ & $\tau_{12}[\mathrm{MPa}]$ & $\sigma_{22}[\mathrm{MPa}]$ \\
\hline-7.50 & 0.00 & 100.00 & 0.0000 & 100.00 \\
\hline-2.50 & 0.00 & 100.00 & 0.0000 & 100.00 \\
\hline 0.00 & 0.00 & 100.00 & 0.0000 & 100.00 \\
\hline 5.00 & 7.50 & 99.999 & 0.0000 & 100.00 \\
\hline 9.00 & 1.00 & 100.00 & 0.0000 & 100.00 \\
\hline
\end{tabular}

Problem 2. We consider an outer region which includes two circular holes with radius $R=10 \mathrm{~mm}$ and a constant stress state $\sigma_{11}(\infty)=100[\mathrm{MPa}], \tau_{12}(\infty)=\tau_{21}(\infty)=$ $\sigma_{22}(\infty)=0$ is prescribed at infinity. The material parameters are the same as those for the first problem. 


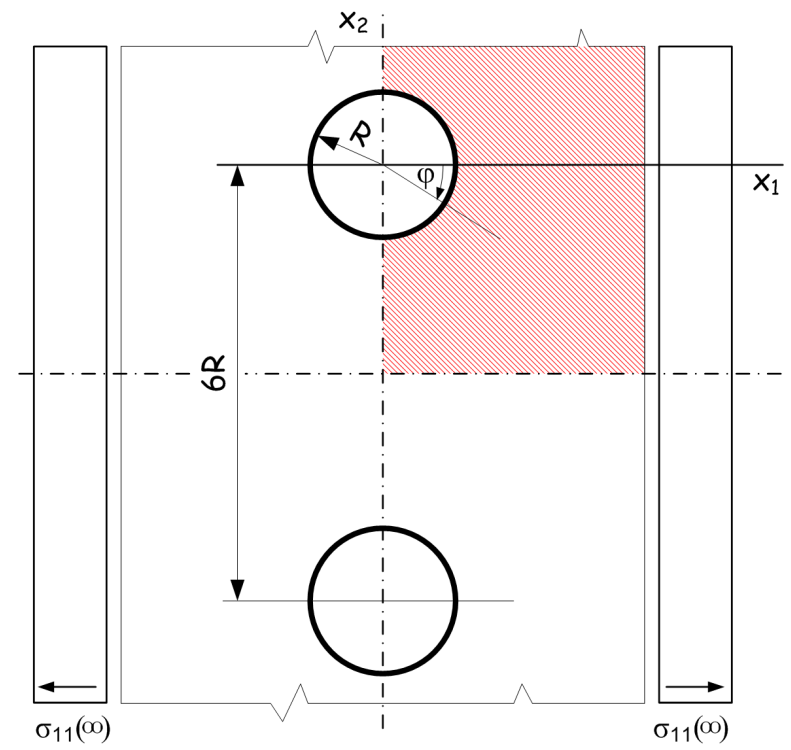

Figure 3. An outer region with two circular holes

In order to validate our numerical solutions for the stress distribution around the two circular holes, we have set up a finite element (FE) model utilizing the symmetry of the problem. The cross-hatched area in Figure 3 indicates the investigated area.
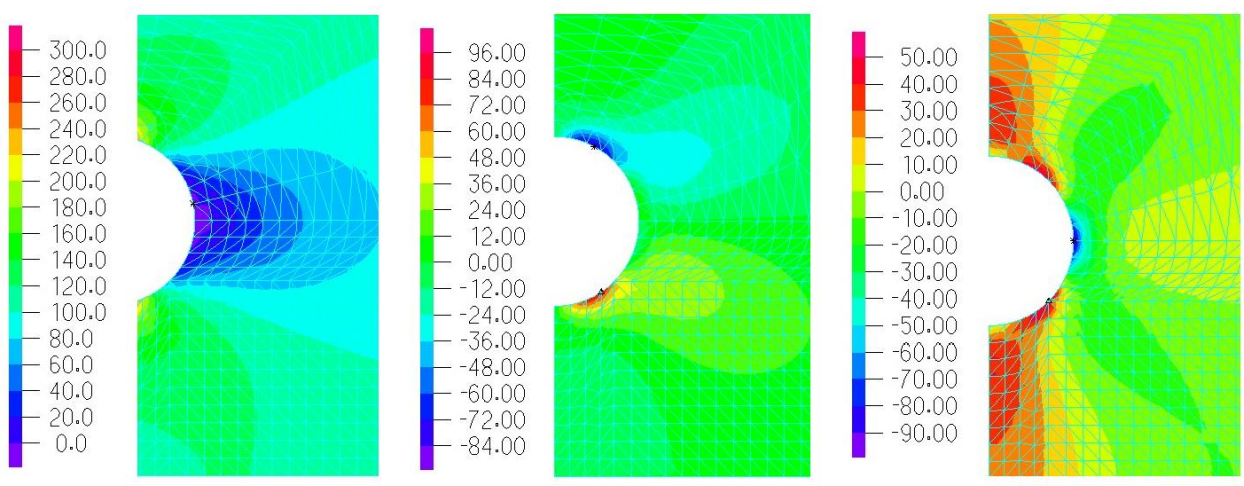

Figure 4. Normal stress $\sigma_{11}$, shear stress $\tau_{12}$ and normal stress $\sigma_{22}$ distributions around the upper hole

The finite element analysis results of ADINA can be seen in Figure 4, where only the stress distributions around the upper circle are presented. 
Table 2: Solutions for stress components along the axes $x_{1}, x_{2}$

\begin{tabular}{|c|c|c|c|c|}
\hline$x_{1}[\mathrm{~mm}]$ & $x_{2}[\mathrm{~mm}]$ & $\sigma_{11}[\mathrm{MPa}]$ & $\tau_{12}[\mathrm{MPa}]$ & $\sigma_{22}[\mathrm{MPa}]$ \\
\hline 0.00 & 20.00 & $121.26(122.87)$ & $0.00(0.47)$ & $27.97(30.03)$ \\
\hline 0.00 & 15.00 & $150.31(153.27)$ & $0.00(0.16)$ & $36.53(37.21)$ \\
\hline 0.00 & 10.00 & $298.60(303.20)$ & $0.00(-1.57)$ & $0.63(1.75)$ \\
\hline \hline 0.00 & -10.00 & $301.02(302.43)$ & $0.00(1.42)$ & $0.57(-0,19)$ \\
\hline 0.00 & -15.00 & $157.68(154.15)$ & $0.00(0.28)$ & $42.26(39.06)$ \\
\hline 0.00 & -20.00 & $127.17(126.91)$ & $0.00(0.10)$ & $36.76(35.66)$ \\
\hline 0.00 & -25.00 & $117.85(117.46)$ & $0.00(0.11)$ & $31.85(29.36)$ \\
\hline 0.00 & -30.00 & $115.55(115.25)$ & $0.00(0.15)$ & $30.23(27.74)$ \\
\hline 0.00 & -35.00 & $117.84(117.46)$ & $0.00(0.11)$ & $31.85(29.36)$ \\
\hline 0.00 & -40.00 & $127.16(126.91)$ & $0.00(0.10)$ & $36.76(35.66)$ \\
\hline 0.00 & -45.00 & $157.68(154.15)$ & $0.00(0.28)$ & $42.26(39.06)$ \\
\hline 0.00 & -50.00 & $300.91(302.43)$ & $0.00(1.42)$ & $0.55(-0,19)$ \\
\hline \hline 10.00 & 0.00 & $0.58(0.65)$ & $0.028(-1.27)$ & $-96.85(-93.14)$ \\
\hline 20.00 & 0.00 & $49.88(48.04)$ & $3.32(1.7)$ & $5.46(5.16)$ \\
\hline 50.00 & 0.00 & $92.92(94.62)$ & $0.58(0.73)$ & $-0.099(-0.025)$ \\
\hline 100.00 & 0.00 & $97.85(97.77)$ & $-0.66(-0.84)$ & $-0.87(-0.84)$ \\
\hline
\end{tabular}

The numerical results in Table 2 are in good agreement with those of the finite element analysis results which appear in round brackets.

\section{Concluding Remarks}

In contrast to the conventional boundary element method, there is a possibility for considering plane problems in a dual formulation. Then the equation system to be solved involves the representation of stresses in terms of stress functions of order one, Hooke's law and the compatibility equations. If the region is multiply connected and/or there is more than one arc on the contour with traction boundary conditions then the compatibility conditions are to be supplemented with further conditions of single valuedness. These are referred to as supplementary compatibility conditions (for single arcs) or compatibility conditions at large (for a whole contour). If the region under consideration is a simply connected one then the boundary element method as well as the boundary contour method have already been worked out in $1,2,9,13$. The present paper is concerned with the generalization of the boundary contour method for multiply-connected regions. For these regions the traction boundary conditions in terms of stress functions of order one contain undetermined constants of integration which can be determined if and only if the field equations are associated with the compatibility conditions at large and/or the supplementary compatibility conditions. In this way one can ensure that the boundary value is determinate. To achieve this goal, we also had to apply those mathematical parameters which are used in the derivation of the $\mathrm{BCM}$ equations when giving the compatibility conditions in a discretized form. Accordingly, the corresponding potential functions, which belong to the supplementary conditions of single valueness or the compatibility conditions 
at large have been determined, provided that the approximation is quadratic. From these potential functions, we have established the discretized forms of the supplementary conditions of single valuedness on the arcs, which are subjected to tractions and the discretized forms of the compatibility condition at large. A program has been developed in Fortran 90 for the numerical investigations. We have found that the displacement derivatives could be replaced by the corresponding compatibility conditions on closed boundary curves. The examples shown represent the applicability of the algorithm to a simple and a bit more difficult problem.

Acknowledgements. The described work was carried out as part of the TÁMOP-4.2.1.B10/2/KONV-2010-0001 project in the framework of the New Hungarian Development Plan. The realization of this project is supported by the European Union, co-financed by the European Social Fund.

\section{REFERENCES}

1. Szirbik, S.: Boundary contour method for plane problems in a dual formulation with linear elements. Journal of Computational and Applied Mechanics, 1(2), (2000), 205-222.

2. Szeidl, G. and Szirbik, S.: Boundary Contour Method for Plane Problems in a Dual Formulation with Quadratic Shape Functions, In: V. Kompis (ed) Selected Topics in Boundary Integral Formulations for Solids and Fluids, Internatiponal Centre for Mechanical Sciences, Volume 433, Springer, 2002, 209-232.

3. Nagarjan, A., Lutz, E. D., and Mukherjee, S.: A novel boundary element method for linear elasticity with no numerical integration for two dimensional and line integrals for three-dimensional problems. Journal of Applied Mechanics, 61, (1994), 264-269.

4. Nagarjan, A., Lutz, E. D., and Mukherjee, S.: The boundary contour method for three-dimensional linear elasticity. Journal of Applied Mechanics, 63, (1996), 278-286.

5. Phan, A. V., Mukherjee, S., and Mayer, J. R. R.: The boundary contour method for two-dimensional linear elasticity with quadratic boundary elements. Computational Mechanics, 20, (1997), 310-319.

6. Phan, A. V., Mukherjee, S., and Mayer, J. R. R.: Stresses, stress sensitivities and shape optimization in two dimensional linear elasticity by the boundary contour method. International Journal for Numerical Methods in Engineering, 42, (1998), 1391-1407.

7. Phan, A. V., Mukherjee, S., and Mayer, J. R. R.: The hypersingular boundary contour method for two-dimensional linear elasticity. Acta Mechanica, 130.

8. SzEIDL, G.: Dual problems of continuum mechanics (derivation of defining equations, single valuedness of mixed boundary value problems, boundary element method for plane problems). Habilitation Booklets of the Miskolc University (in Hungarian), Faculty of Mechanical Engineering, University of Miskolc, Department of Mechanics, 1997.

9. SzeidL, G.: Boundary integral equations for plane problems in terms of stress functions of order one. Journal of Computational and Applied Mechanics, 2(2), (2001), 237-261.

10. SzeidL, G.: On compatibility conditions for mixed boundary value problems. Technische Mechanik, 17, (1997), 245-262.

11. Szeidu, G.: Problems of Continuum Mechanics in a Dual Formulation (Derivation of the definig equations, Single valudness of mixed boundary value problems, Boundary element 
method for plane problems). Thesis for the degree Doctor of Science awarded by the Hungarian Academy of Sciences (in Hungarian), 2004.

12. SZIRBIK, S.: Boundary contour method for plane problems in the dual system of elasticity. $\mathrm{PhD}$ dissertation (in Hungarian), Faculty of Mechanical Engineering, University of Miskolc, Department of Mechanics, 2003.

13. SzIrbik, S.: Boundary contour method for plane problems with linear elements in dual system. In microCAD-SYSTEM '2000 International Computer Science Conference, ME Miskolc, 2000, pp. 157-162.

ApPEndix A.

A.1. The fundamental solutions of order one and two.

$$
\left[\mathfrak{U}_{k l}(M, Q)\right]=\frac{\mu}{4 \pi(1-\nu)}\left[\begin{array}{ccc}
-2 \ln R-3-2 \frac{r_{2} r_{2}}{R^{2}} & 2 \frac{r_{1} r_{2}}{R^{2}} & \frac{2}{\mu}(1-\nu) \frac{r_{1}}{R^{2}} \\
2 \frac{r_{2} r_{1}}{R^{2}} & -2 \ln R-3-2 \frac{r_{1} r_{1}}{R^{2}} & \frac{2}{\mu}(1-\nu) \frac{r_{2}}{R^{2}} \\
\frac{2}{\mu}(1-\nu) \frac{r_{1}}{R^{2}} & \frac{2}{\mu}(1-\nu) \frac{r_{2}}{R^{2}} & 0
\end{array}\right]
$$

where $\mathfrak{D}(R)=1 / 8 \pi(1-\nu) R^{2}$ and the normal $n_{\rho}$ is taken at the point $M$. The distance between the points $M$ and $Q$ is $R$, which is defined as absolute value of the vector $r_{\rho}=x_{\rho}(M)-x_{\rho}(Q)$.

The matrix $\mathfrak{T}_{1 \lambda \rho}$ is derived by factoring out the outward normal $n_{1}(M)$ from A.1b as follows:

$$
\left[\mathfrak{T}_{1 \lambda \rho}\right]=\mathfrak{D}(R)\left[\begin{array}{cc}
-2(3-2 \nu) \eta_{1}+\frac{4 \eta_{2}^{2} \eta_{1}}{\eta_{1}^{2}+\eta_{2}^{2}} & -2(3-2 \nu) \eta_{2}+\frac{4 \eta_{2}^{3}}{\eta_{1}^{2}+\eta_{2}^{2}} \\
2(1-2 \nu) \eta_{2}-\frac{4 \eta_{1}^{2} \eta_{2}}{\eta_{1}^{2}+\eta_{2}^{2}} & -2(1-2 \nu) \eta_{1}-\frac{4 \eta_{2}^{2} \eta_{1}}{\eta_{1}^{2}+\eta_{2}^{2}}
\end{array}\right]
$$

The matrix $\mathfrak{T}_{2 \lambda \rho}$ is also derived by factoring out the outward normal $n_{2}(M)$ from A.1b as follows:

$$
\left[\mathfrak{T}_{2 \lambda \rho}\right]=\mathfrak{D}(R)\left[\begin{array}{cc}
-2(1-2 \nu) \eta_{2}-\frac{4 \eta_{1}^{2} \eta_{2}}{\eta_{1}^{2}+\eta_{2}^{2}} & 2(1-2 \nu) \eta_{1}-\frac{4 \eta_{1} \eta_{2}^{2}}{\eta_{1}^{2}+\eta_{2}^{2}} \\
-2(3-2 \nu) \eta_{1}+\frac{4 \eta_{1}^{3}}{\eta_{1}^{2}+\eta_{2}^{2}} & -2(3-2 \nu) \eta_{2}+\frac{4 \eta_{1}^{2} \eta_{2}}{\eta_{1}^{2}+\eta_{2}^{2}}
\end{array}\right]
$$


Appendix B.

B.1. Quadratic shape functions. If the approximation is quadratic, the shape functions are as follows:

$$
\begin{aligned}
& { }^{1} \phi_{1}=\frac{1}{2 \pi} \arctan \frac{\eta_{2}}{\eta_{1}}+\frac{1}{4 \pi(1-\nu)} \frac{\eta_{1} \eta_{2}}{\eta_{1}^{2}+\eta_{2}^{2}}, \\
& { }^{2} \phi_{1}=-\frac{\eta_{2}}{4 \pi(1-\nu)}\left\{\ln \sqrt{\eta_{1}^{2}+\eta_{2}^{2}}+\frac{4 \nu-3}{2}+\frac{2 \eta_{2}^{2}}{\eta_{1}^{2}+\eta_{2}^{2}}\right\}, \\
& { }^{3} \phi_{1}=\frac{\eta_{1}}{4 \pi(1-\nu)}\left\{(1-\nu) \ln \sqrt{\eta_{1}^{2}+\eta_{2}^{2}}+\frac{3-\nu}{2}-\frac{\eta_{1}^{2}}{\eta_{1}^{2}+\eta_{2}^{2}}\right\}, \\
& { }^{4} \phi_{1}=-\frac{1}{4 \pi(1-\nu)}\left\{(1-2 \nu) \ln \sqrt{\eta_{1}^{2}+\eta_{2}^{2}}+\frac{\eta_{1}^{2}}{\eta_{1}^{2}+\eta_{2}^{2}}\right\}, \\
& { }^{5} \phi_{1}=\frac{\eta_{1}}{4 \pi(1-\nu)}\left\{\nu \ln \sqrt{\eta_{1}^{2}+\eta_{2}^{2}}+\frac{5 \nu}{2}-\frac{\eta_{1}^{2}}{\eta_{1}^{2}+\eta_{2}^{2}}\right\}, \\
& { }^{6} \phi_{1}=-\frac{\mu \eta_{2}}{4 \pi(1-\nu)}\left\{2 \ln \sqrt{\eta_{1}^{2}+\eta_{2}^{2}}+3\right\}, \\
& { }^{7} \phi_{1}=\frac{1}{8 \pi(1-\nu)}\left\{2(\nu-1) \eta_{1}^{2}+(1-2 \nu) \eta_{2}^{2}+\right. \\
& \left.+\left[(\nu-1) \eta_{1}^{2}+(2-\nu) \eta_{2}^{2}\right] \ln \left(\eta_{1}^{2}+\eta_{2}^{2}\right)+\frac{6 \eta_{2}^{4}}{\eta_{1}^{2}+\eta_{2}^{2}}\right\}, \\
& { }^{8} \phi_{1}=\frac{1}{4 \pi(1-\nu)}\left\{\left[3-5 \nu-\nu \ln \left(\eta_{1}^{2}+\eta_{2}^{2}\right)\right] \eta_{1} \eta_{2}-\frac{3 \eta_{1} \eta_{2}^{3}}{\eta_{1}^{2}+\eta_{2}^{2}}\right\}, \\
& { }^{9} \phi_{1}=\frac{1}{4 \pi(1-\nu)}\left\{\left[4-3 \nu+(1-\nu) \ln \left(\eta_{1}^{2}+\eta_{2}^{2}\right)\right] \eta_{1} \eta_{2}-\frac{\eta_{1}^{3} \eta_{2}}{\eta_{1}^{2}+\eta_{2}^{2}}\right\}, \\
& { }^{10} \phi_{1}=\frac{1}{8 \pi(1-\nu)}\left\{(1+4 \nu) \eta_{1}^{2}+(4-4 \nu) \eta_{2}^{2}+\left[(1-\nu) \eta_{2}^{2}+\nu \eta_{1}^{2}\right] \ln \left(\eta_{1}^{2}+\eta_{2}^{2}\right)-\frac{2 \eta_{1}^{4}}{\eta_{1}^{2}+\eta_{2}^{2}}\right\}, \\
& { }^{1} \phi_{2}=\frac{1}{4 \pi(1-\nu)}\left\{(1-2 \nu) \ln \sqrt{\eta_{1}^{2}+\eta_{2}^{2}}+\frac{\eta_{2}^{2}}{\eta_{1}^{2}+\eta_{2}^{2}}\right\}, \\
& { }^{2} \phi_{2}=-\frac{\eta_{1}}{4 \pi(1-\nu)}\left\{\ln \sqrt{\eta_{1}^{2}+\eta_{2}^{2}}+\frac{4 \nu-3}{2}+\frac{2 \eta_{1}^{2}}{\eta_{1}^{2}+\eta_{2}^{2}}\right\}, \\
& { }^{3} \phi_{2}=-\frac{\eta_{2}}{4 \pi(1-\nu)}\left\{\nu \ln \sqrt{\eta_{1}^{2}+\eta_{2}^{2}}+\frac{5 \nu}{2}-\frac{\eta_{2}^{2}}{\eta_{1}^{2}+\eta_{2}^{2}}\right\}, \\
& { }^{4} \phi_{2}=-\frac{1}{2 \pi} \arctan \frac{\eta_{1}}{\eta_{2}}-\frac{1}{4 \pi(1-\nu)} \frac{\eta_{1} \eta_{2}}{\eta_{1}^{2}+\eta_{2}^{2}}, \\
& { }^{5} \phi_{2}=-\frac{\eta_{2}}{4 \pi(1-\nu)}\left\{(1-\nu) \ln \sqrt{\eta_{1}^{2}+\eta_{2}^{2}}+\frac{3-\nu}{2}-\frac{\eta_{2}^{2}}{\eta_{1}^{2}+\eta_{2}^{2}}\right\}, \\
& { }^{6} \phi_{2}=\frac{\mu \eta_{1}}{4 \pi(1-\nu)}\left\{2 \ln \sqrt{\eta_{1}^{2}+\eta_{2}^{2}}+3\right\} \\
& { }^{7} \phi_{2}=\frac{1}{4 \pi(1-\nu)}\left\{\left[5 \nu-3+\nu \ln \left(\eta_{1}^{2}+\eta_{2}^{2}\right)\right] \eta_{1} \eta_{2}+\frac{3 \eta_{1}^{3} \eta_{2}}{\eta_{1}^{2}+\eta_{2}^{2}}\right\}, \\
& { }^{8} \phi_{2}=\frac{1}{8 \pi(1-\nu)}\left\{(2 \nu-1) \eta_{1}^{2}+2(1-\nu) \eta_{2}^{2}+\right.
\end{aligned}
$$




$$
\begin{gathered}
\left.+\left[(1-\nu) \eta_{2}^{2}+(\nu-2) \eta_{1}^{2}\right] \ln \left(\eta_{1}^{2}+\eta_{2}^{2}\right)-\frac{6 \eta_{1}^{4}}{\eta_{1}^{2}+\eta_{2}^{2}}\right\} \\
{ }^{9} \phi_{2}=\frac{1}{8 \pi(1-\nu)}\left\{(4 \nu-4) \eta_{1}^{2}-(1+4 \nu) \eta_{2}^{2}+\left[(\nu-1) \eta_{1}^{2}-\nu \eta_{2}^{2}\right] \ln \left(\eta_{1}^{2}+\eta_{2}^{2}\right)+\frac{2 \eta_{2}^{4}}{\eta_{1}^{2}+\eta_{2}^{2}}\right\} \\
{ }^{10} \phi_{2}=\frac{1}{4 \pi(1-\nu)}\left\{\left[3 \nu-4-(1-\nu) \ln \left(\eta_{1}^{2}+\eta_{2}^{2}\right)\right] \eta_{1} \eta_{2}+\frac{\eta_{1} \eta_{2}^{3}}{\eta_{1}^{2}+\eta_{2}^{2}}\right\}
\end{gathered}
$$

Appendix C.

C.1. The transformation matrix $\mathbf{B}^{Q}$. If the approximation is quadratic, the matrix $\mathbf{B}^{Q}$ is as follows:

$$
\begin{aligned}
& {\left[\mathbf{B}^{Q}\right]=\left[\begin{array}{cccccc}
1 & x_{1}(Q) & x_{2}(Q) & 0 & 0 & 0 \\
0 & 1 & 0 & 0 & 0 & 0 \\
0 & 0 & 1 & 0 & 0 & 0 \\
0 & -x_{2}(Q) & 0 & 1 & x_{1}(Q) & 0 \\
0 & 0 & 0 & 0 & 1 & 0 \\
0 & 0 & 0 & 0 & 0 & 1 \\
0 & 0 & 0 & 0 & 0 & 0 \\
0 & 0 & 0 & 0 & 0 & 0 \\
0 & 0 & 0 & 0 & 0 & 0 \\
0 & 0 & 0 & 0 & 0 & 0
\end{array}\right.} \\
& -2 x_{1}(Q) x_{2}(Q) \\
& -2 x_{2}(Q) \\
& -2 x_{1}(Q) \\
& 2 x_{1}(Q) \\
& x_{2}^{2}(Q) \quad 0 \\
& x_{2}^{2}(Q) \\
& 0 \\
& 0 \quad 0 \\
& 0 \\
& -2 x_{1}(Q) x_{2}(Q) \\
& 2 x_{2}(Q) \quad 0 \\
& 0 \\
& -2 x_{2}(Q) \\
& 0 \quad x_{1}^{2}(Q) \\
& K x_{2}(Q) \\
& K x_{1}(Q) \quad K x_{1}(Q) \quad K x_{2}(Q) \\
& 1 \\
& 0 \\
& 0 \quad 0 \\
& 0 \\
& 1 \\
& 0 \quad 0 \\
& 0 \\
& 0 \\
& 10 \\
& 0 \\
& 0 \\
& 0 \\
& 1
\end{aligned}
$$

0
0
0
1

